\title{
A New Class of Exactly Solvable Models within the Schrödinger Equation with Position Dependent Mass
}

\author{
Anis Dhahbi ${ }^{1}$, Yassine Chargui ${ }^{1}$, Adel Trablesi ${ }^{2}$ \\ ${ }^{1}$ Physics Department, College of Science and Arts at ArRass, Qassim University, Al-Mulida, KSA \\ ${ }^{2}$ Université de Tunis El Manar, Faculté des Sciences de Tunis, Unité de Recherche de Physique Nucléaire et des \\ Hautes Energies, Tunis, Tunisie \\ Email: anis.dhahbi14@gmail.com, yassine.chargui@gmail.com, adel.trabelsi@gmail.com
}

How to cite this paper: Dhahbi, A., Chargui, Y. and Trablesi, A. (2019) A New Class of Exactly Solvable Models within the Schrödinger Equation with Position Dependent Mass. Journal of Applied Mathematics and Physics, 7, 1013-1026.

https://doi.org/10.4236/jamp.2019.75068

Received: April 3, 2019

Accepted: May 3, 2019

Published: May 6, 2019

Copyright (c) 2019 by author(s) and Scientific Research Publishing Inc.

This work is licensed under the Creative Commons Attribution International License (CC BY 4.0).

http://creativecommons.org/licenses/by/4.0/

\section{Open Access}

\begin{abstract}
The study of physical systems endowed with a position-dependent mass (PDM) remains a fundamental issue of quantum mechanics. In this paper we use a new approach, recently developed by us for building the quantum kinetic energy operator (KEO) within the Schrödinger equation, in order to construct a new class of exactly solvable models with a position varying mass, presenting a harmonic-oscillator-like spectrum. To do so we utilize the formalis$\mathrm{m}$ of supersymmetric quantum mechanics (SUSY QM) along with the shape invariance condition. Recent outcomes of non-Hermitian quantum mechanics are also taken into account.
\end{abstract}

\section{Keywords}

Schrödinger Equation, Position Dependent Mass, Kinetic Energy Operator, Solvable Models, Supersymmetric Quantum Mechanics, Shape Invariance

\section{Introduction}

The idea of non-relativistic or relativistic particle whose mass is a function of space becomes an intensive field of research for the past few decades. Indeed, there are many problems in physics, chemistry, biology and even in medicine where the evolution of a phenomenon can be assimilated by a Schrödinger, Klein-Gordon or even Dirac type equation, relative to a particle of variable mass in space. Especially, in the case of solid-state physics, the movement of a particle in a periodic potential representing the crystalline lattice, is assimilated to the movement of a free particle with an effective mass, which depends essentially on the characteristics of the lattice. If the sample 
is composed of several parts representing different materials, called heterostructures, the mass will take different values in each part of the structure. Accordingly, Position Dependent Mass (PDM) model$\mathrm{s}$ [1-12] were successfully applied in the determination of electronic properties of semiconductors [12-16], as well as for the description of the properties of quantum dots [17] and nuclear clusters $[18,19]$. The PDM issue has also attracted an increasing interest in molecular and atomic physics [20,21], density functional theory [2, 5, 6, 22, 23], and even the theory of general relativity, where systems with PDM are described with another origin of creation, different from the condensed matter one [24]. Hence, quantum mechanics theorists continue to investigate the subject of PDM Hamiltonians as a fundamental issue which is still far from being entirely resolved. In addition to the exact solvability [25-32], the PDM problem raises several major conceptual questions, like the boundary and continuity conditions $[11,12,33]$, the Galilean invariance of the theory $[14,33]$ and the implementation of path-integral technique $[34,35]$.

While the interest in searching exactly solvable quantum PDM systems is still increasing $[25,36]$, the most interesting question remains how to order the mass operator with respect to the momentum operator when it comes to building the KEO of the Hamiltonian. The dilemma does not seem obvious when the mass is constant, but when this latter becomes a function of the particle position, the bewilderment appears in the non-commutativity between the mass and the momentum operators. In other words, when a particle is endowed with a variable mass (position-dependent mass), this latter will not commute with the particle's momentum operator anymore. Thus, the Hermiticity condition of the Hamiltonian, seems to be inadequate to definitely specify a unique form for the Kinetic Energy Operator (KEO) with a position-dependent effective mass. Such a dilemma brings us back to rethink about the general rule has to be followed in associating operators in quantum-mechanical with respect to the classical quantities (see for example Ref. [37]). For this purpose, many criteria have been adopted in order to define the right form of the KEO, such as the current-density conservation and the agreement between theoretical and experimental results, but none of the proposed forms of KEO's has been hitherto able to provide a clear-cut answer to this question. In this regard, Ref. [38] can be reviewed to check more details out about that issue. Moreover, many contributions to this subject are also worthy of note, such as BenDaniel and Duke [7], Zhu and Kroemer [11], Dutra and Almeida [31], Gora and Williams [10], Von Roos [14], Trabelsi et al. [38] and Morrow and Brownstein [12]. Therein, several forms of the KEO are shown to be useful in lot of applications, as for example Li-Kuhn [8], BenDaniel and Duke [2], and so on. However, the common denominator of all these researches is the portrayal of the KEO's as different orderings of powers of the mass and the momentum operators.

But on the other hand, it was proved by C. Bender works [39-47] that there is no physical reason to restrict quantum mechanics to Hermitian Hamiltonian only. Although, this work proposes to explore other uncustomary ways to build the quantum Hamiltonian KEO of a PDM system with more simplicity and generality. In fact, we have recently developed a novel approach for constructing the quantum operator associated to the non-relativistic classical kinetic energy, within the Schrödinger equation [48]. The idea behind lies on the fact that 
the coefficient-functions of the momentum in the KEO, may take, a priori, an arbitrary form. As a matter of fact, in the literature [49-52], these functions have been always assumed to be powers of the varying mass. Moreover, in compliance with recent outcomes of non-Hermitian quantum mechanics, the requirement that the Hamiltonian be Hermitian has been dropped in favor of the pseudo-Hermiticity condition. In this work, we intend to use this method to generate some new solvable models with PDM, on the basis of the formalism of supersymmetric quantum mechanics (SUSY QM) and the notion of shape invariance.

The paper is organized as follows: in Section 1, we outline the new way we shall use to approach the problem of a quantum Hamiltonian with PDM. In Section 2, we employ the SUSY approach relative to this Hamiltonian along with the shape invariance condition, in order to generate a class of exactly PDM solvable models with a harmonicoscillator (HO)-like spectrum. In the final section we give our conclusion.

\section{Outlines of the New Approach to PDMSE}

In Ref [48], we have addressed the problem of a one-dimensional PDM Schrödinger equation, by suggesting a KEO $\hat{T}$ having the form:

$$
\hat{T}=\frac{1}{2} \alpha(\hat{x}) \hat{p} \beta(\hat{x}) \hat{p} \gamma(\hat{x})
$$

where $\alpha, \beta$ and $\gamma$ are arbitrary real functions of the position operator, and not necessary powers of the mass function, as it is assumed in the literature so far. But of course, these functions have to verify the condition:

$$
\alpha(x) \beta(x) \gamma(x)=1 / m(x)
$$

where $m(x)$ is the position-dependent mass of the considered system. Then, given a real potential interaction $V(\hat{x})$, the resulting Hamiltonian is in general not Hermitian. As a matter of fact, it has been shown that there is no physical reason to restrict quantum mechanics to Hermitian Hamiltonian only [53-56]. Indeed, a quasi-Hermitian Hamiltonian may brings back to a real spectrum. As proven in [56], such an assumption requires that there exists a Hermitian, positivedefinite and bounded operator $\eta$, allowing to write:

$$
\hat{H}^{\dagger}=\eta \hat{H} \eta^{-1}
$$

where $\hat{H}^{\dagger}$ is the standard Hermitian adjoint of $\hat{H}$. Hence, considering an operator $\eta(x)=\gamma(x) / \alpha(x)$ allows to fulfill the condition (3), and makes $\hat{H}$ quasi-Hermitian, provided that $\eta$ is bounded and $\alpha(x)$ and $\gamma(x)$ have the same sign. In that case, it will be possible to relate the original $\hat{H}$ to a Hermitian counterpart, let it be $\hat{H}_{h}$, satisfying:

$$
\hat{H}_{h}=\eta^{1 / 2} \hat{H} \eta^{-1 / 2}=\frac{1}{2} \omega(\hat{x}) \hat{p} \beta(\hat{x}) \hat{p} \omega(\hat{x})+V(\hat{x})
$$

with $\omega=\sqrt{\alpha \gamma}$. Eventually, $\hat{H}$ and $\hat{H}_{h}$ are iso-spectral, and the eigenvectors $|\psi\rangle$ of the Hamiltonian $\hat{H}$ will be expressed as a function of the solutions $\left|\psi_{h}\right\rangle$ of the Hamiltonian $\hat{H}_{h}$ as:

$$
|\psi\rangle=\eta^{-1 / 2}\left|\psi_{h}\right\rangle
$$


At this regard, the Hamiltonian $\hat{H}_{h}$ can be factorized using two intertwining operators, $\hat{A}$ and $\hat{A}^{\dagger}$ as

$$
\hat{H}_{h}=\hat{A}^{\dagger} \hat{A}+E_{0}
$$

where $E_{0}$ is the ground-state energy of $\hat{H}_{h}$ and $\hat{A}$ and $\hat{A}^{\dagger}$ are defined by

$$
\hat{A}=\frac{1}{\sqrt{2}} \sqrt{\beta(\hat{x})} \hat{p} \omega(\hat{x})-i W(\hat{x}), \quad \hat{A}^{\dagger}=\frac{1}{\sqrt{2}} \omega(\hat{x}) \hat{p} \sqrt{\beta(\hat{x})}+i W(\hat{x})
$$

with $W(\hat{x})$ the corresponding real superpotential. Thus we can establish that

$$
\hat{H}_{2}=\hat{H}_{1}+\frac{\hbar^{2}}{2 \sqrt{m(\hat{x})}}\left\{\frac{W^{\prime}(\hat{x})}{W(\hat{x}) \sqrt{m(\hat{x})}}+\frac{\sqrt{2}}{\hbar}\left[\frac{\tilde{V}(\hat{x})}{W(\hat{x})}+W(\hat{x})\right]\right\}^{\prime}
$$

where $\hat{H}_{1}$ and $\hat{H}_{2}$ are the SUSY partner Hamiltonians, defined as

$$
\hat{H}_{1}=\hat{H}_{h}=\hat{A}^{\dagger} \hat{A}+E_{0} \quad, \quad \hat{H}_{2}=\hat{A} \hat{A}^{\dagger}+E_{0}
$$

Then, by imposing the integrability condition within the SUSY QM theory, that is the shape invariance (SI) of the potential $V(x)$, we show that the eigenvalue problem corresponding to the Hamiltonian (4) can solved exactly if the following condition is fulfilled:

$$
\frac{\hbar^{2}}{2 \sqrt{m(x)}}\left\{\frac{W^{\prime}(x)}{W(x) \sqrt{m(x)}}+\frac{\sqrt{2}}{\hbar}\left[\frac{\tilde{V}(x)}{W(x)}+W(x)\right]\right\}^{\prime}=F(x)
$$

with $F(x)$ the function given, according to the SI condition, as

$$
F(x)=V\left(x, f\left(a_{1}\right)\right)-V\left(x, a_{1}\right)+R\left(a_{2}\right)
$$

where $a_{1}$ is in general the sets of parameters of the potential $V(x)$. In addition, the function $\beta(x)$, seen as the free function of the Hamiltonian $\hat{H}_{h}$, is related to the potential $\tilde{V}$ and the superpotential as

$$
\beta(x)=\frac{1}{W(x) \sqrt{m(x)}} \exp \left\{\int^{x} \frac{\sqrt{2 m(y)}}{\hbar}\left[W(y)-\frac{\tilde{V}(y)}{W(y)}\right] d y\right\}
$$

where the integration constant has been set to one and this choice has no restriction. Thus, given a mass function $m(x)$ and a superpotential $W(x)$, Equation (10) can be used to fix the potential $V(x)$, for which the corresponding quantum problem is exactly solvable. Then Equation (12) allows to determine the corresponding KEO. Notice that the ground state wave function $\psi_{0}(x)$, is determined from the equation $\hat{A} \psi_{0}=0$. This would give

$$
\psi_{0}(x)=C \sqrt{\beta(x) m(x)} \exp \left\{-\int^{x} \frac{\sqrt{2 m(y)}}{\hbar} W(y) d y\right\}
$$

with $C$ a normalization constant. And let us recall that $\psi_{0}(x)$ should be normalizable in order to have an unbroken SUSY [57]. On the other hand, we should note that Equation (10) is actually a Riccati equation for $W(x)$, so it can be always transformed to the a second order linear differential equation.

In the next section, we shall start from Equation (10) in order to generate a class of solvable systems having a HO-like spectrum. 


\section{A Class of PDM Systems with a HO-Like Spectrum}

In this section, we shall be interested in the class of systems exhibiting a HO-like spectrum. Such a class corresponds to the following commutation relation:

$$
\left[\hat{A}, \hat{A}^{\dagger}\right]=\kappa
$$

with $\kappa$ a real positive constant. In that case the spectrum of the Hamiltonian $\hat{H}_{h}$ is given as

$$
E_{n}=\sqrt{\kappa} n+E_{0}, \quad n=0,1,2, \ldots
$$

On the other hand, according to Equation (10), this relation means that the potential $\tilde{V}(x)$ can be written as a function of the mass and the superpotential as:

$$
\frac{\hbar^{2}}{2 \sqrt{m(x)}}\left\{\frac{W^{\prime}(x)}{W(x) \sqrt{m(x)}}+\frac{\sqrt{2}}{\hbar}\left[\frac{\tilde{V}(x)}{W(x)}+W(x)\right]\right\}^{\prime}=\kappa
$$

or equivalently

$$
\tilde{V}(x)=-\frac{\hbar}{\sqrt{2 m(x)}} W^{\prime}(x)-W^{2}(x)+\frac{\kappa W(x)}{\hbar} \int^{x} \sqrt{2 m(y)} d y
$$

Furthermore, it is always possible to reexpress the superpotential through a new function $U$ as:

$$
W(x)=\frac{1}{\sqrt{2} \hbar} \int^{x} \sqrt{m(y)}[\kappa+U(y)] d y
$$

Inserting this from in Equation (17) we can readily show that the potential $\tilde{V}(x)$ takes the form

$$
\begin{aligned}
\tilde{V}(x)=-\frac{1}{2}[\kappa+U(x)]+ & \frac{1}{2 \hbar^{2}}\left\{\kappa^{2}\left[\int^{x} \sqrt{m(y)} d y\right]^{2}\right. \\
& \left.-\left[\int^{x} \sqrt{m(y)} U(y) d y\right]^{2}\right\}
\end{aligned}
$$

Now, this equation will be used to generate some concrete models exhibiting a HO-like spectrum.

- Case 1: $m(x)=m_{0}\left(1+a x^{2}\right)$

With $m_{0}$ a constant mass and $a$ a parameter which characterizes the deviation from the usual HO. This form of the mass has been extensively studied in the literature, as it is relevant for the description of the $G a A s / A l_{x} G a_{1-x} A_{s}$ system [58]. Now by choosing

$$
U(x)=\frac{\kappa a x^{2}}{1+a x^{2}}
$$

we find

$$
V(x)=-\frac{\kappa a x^{2}}{2\left(1+a x^{2}\right)}+\frac{\kappa^{2}}{2 \hbar^{2} \sqrt{a}} x \sqrt{1+a x^{2}}(\sqrt{a} x)
$$

where we have omitted the constant term of the potential. This potential is roughly a double-well potential, thus it may have applications 
in molecular physics, for instance. The variations of the potential (21) are shown in Figure 1 for some illustrative values of the free potential parameter. Another interesting potential can be obtained by taking

$$
U(x)=\frac{\kappa / 2}{1+a x^{2}}
$$

This gives

$$
\begin{array}{r}
V(x)=-\frac{\kappa}{2\left(1+a x^{2}\right)}+\frac{\kappa^{2}}{8 \hbar^{2}}\left[x^{2}\left(1+a x^{2}\right)\right. \\
\left.+\frac{2 x}{\sqrt{a}} \sqrt{1+a x^{2}}(\sqrt{a} x)\right]
\end{array}
$$

The last potential is sketched in Figure 2 for two illustrative values of its free parameter.

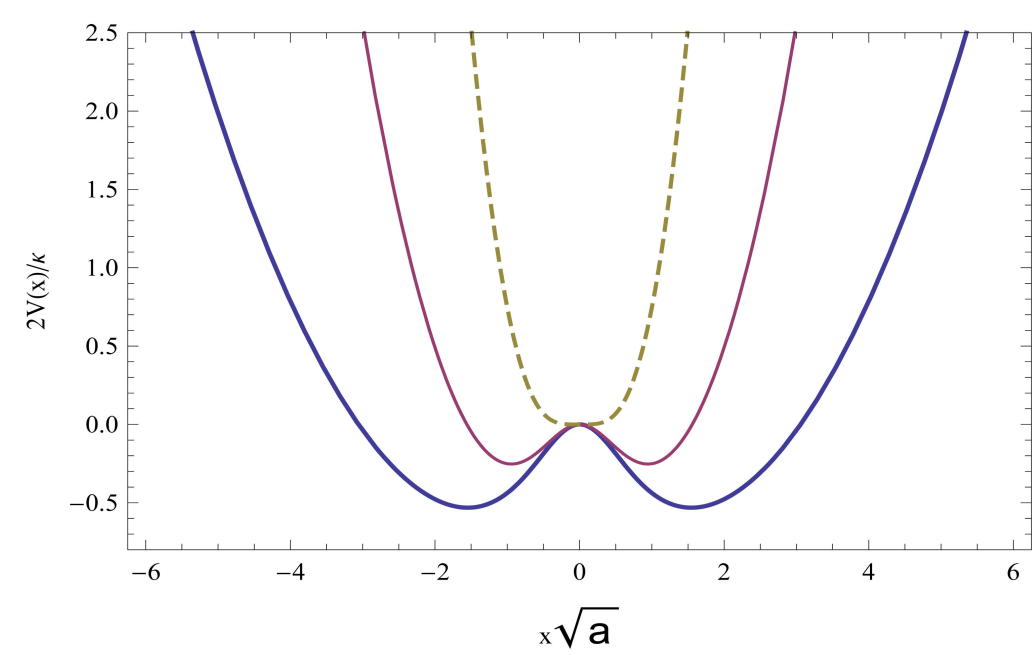

Figure 1. The potential (21) in units of $\kappa / 2$, as a function of $\sqrt{a} x$ for $\kappa / \hbar^{2} a=0.05$ (thick line), $\kappa / \hbar^{2} a=0.2$ (thin line) and $\kappa / \hbar^{2} a=1$ (dashed line).

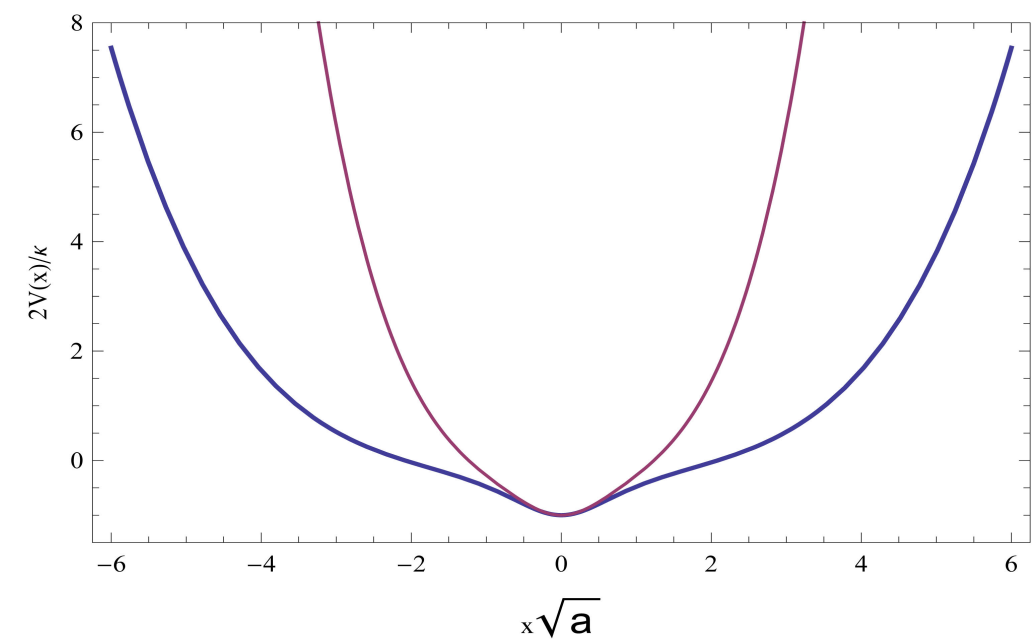

Figure 2. The potential (23) in units of $\kappa / 2$, as a function of $\sqrt{a} x$ for $\kappa / \hbar^{2} a=0.02$ (thick line) and $\kappa / \hbar^{2} a=0.5$ (thin line). 
- Case 2: $m(x)=m_{0} /\left(1+a x^{2}\right)$ with $a>0$

Here $m_{0}$ is a constant mass. This form recalls the MathewsLakshmanan (ML) oscillator, a model which is attracting considerable interest recent years (see for instance $[59,60]$ ). Then by putting $U(x)=0$, we construct the following potential.

$$
V(x)=\frac{m_{0} \kappa^{2}}{2 a \hbar^{2}}(\sqrt{a} x)
$$

For $a x \ll 1$ this potential behaves like a harmonic potential. Thus it may be used in physical situations when additional nonharmonic terms should be included. The variations of the potential (21) are represented in Figure 3 for two illustrative values of its free parameter.

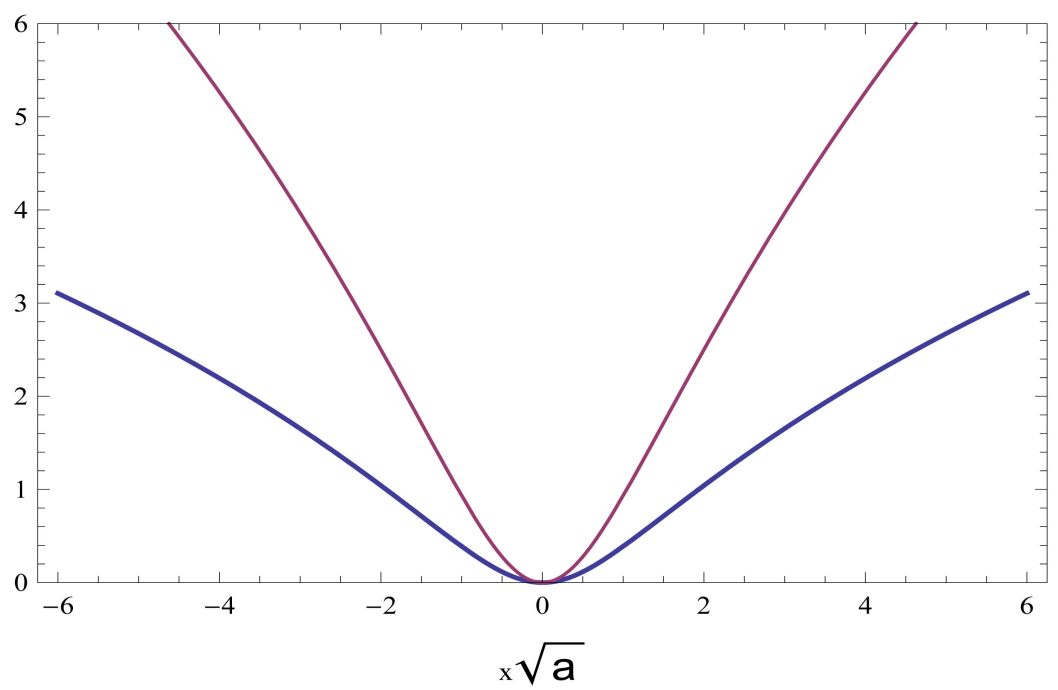

Figure 3. The potential (24) in units of $\kappa$, as a function of $\sqrt{a} x$ for $\frac{m_{0} \kappa}{a \hbar^{2}}=1$ (thick line) and $\frac{m_{0} \kappa}{a \hbar^{2}}=2.4$ (thin line).

Let also point out that when $U(x)=0$, Equations $(18,19)$, yield

$$
V(x)=W^{2}(x)-\frac{\kappa}{2}
$$

and by virtue of Equation (12), this leads to $\beta(x)=1 / \sqrt{m(x)}$. Hence, the resulting KEO corresponds to Li-Kuhn ordering [8], that is:

$$
\hat{T}=\frac{1}{2} \hat{m}^{-1 / 4} \hat{p} \frac{1}{\sqrt{\hat{m}}} \hat{p} \hat{m}^{-1 / 4}
$$

- Case 3: $m(x)=m_{0} e^{2 a x} /\left(1+e^{a x}\right)^{4}$

Here $m_{0}$ is a constant mass and $a$ a real parameter. Such kind of mass distribution is extensively used in the physics of semiconductor quantum well structures [61], and may have applications in molecular physics [62]. Then by choosing

$$
U(x)=\frac{b}{1+e^{a x}}
$$

we obtain the following potential

$$
V(x)=\frac{-b}{2\left(1+e^{a x}\right)}+\frac{m_{0}}{2 a^{2} \hbar^{2}\left(1+e^{a x}\right)^{2}}\left[\kappa^{2}-\frac{b^{2}}{4\left(1+e^{a x}\right)^{2}}\right]
$$


Such type of potential is very relevant especially for studies in molecular physics. In particular for $b=0$, i.e. $U(x)=0$ it reduces to a generalized Woods-Saxon potential. Figure 4 shows the variations of potential (28) for some illustrative values of its free parameters.

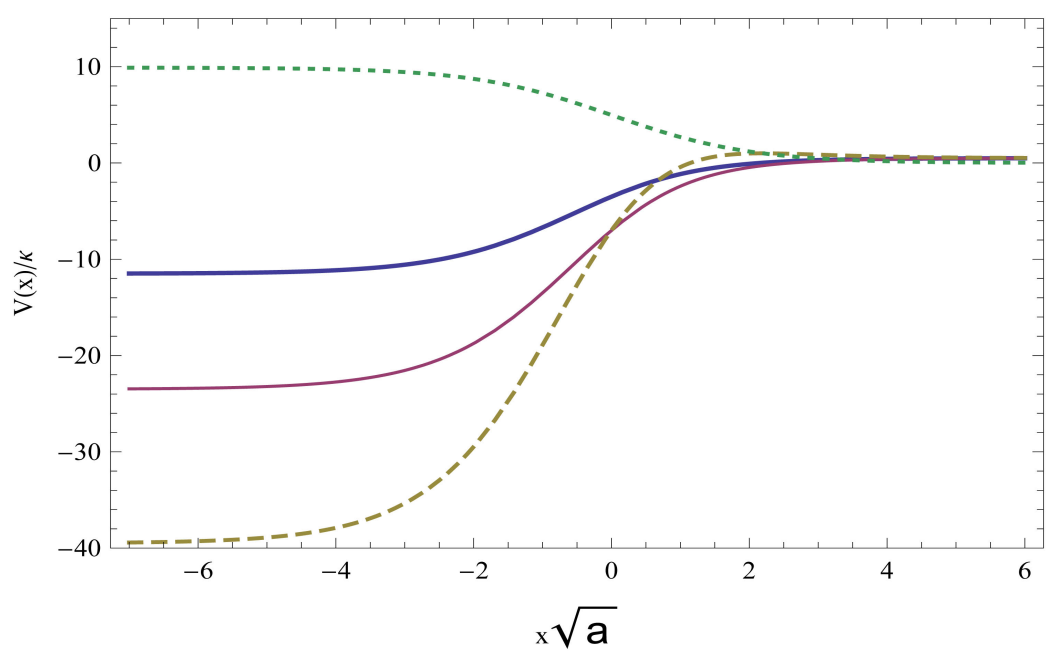

Figure 4. The potential (28) in units of $\kappa$, as a function of $\sqrt{a} x$ for $b / \kappa=8$ and $\frac{m_{0} \kappa}{a^{2} \hbar^{2}}=1$ (thick line), $b / \kappa=12$ and $\frac{m_{0} \kappa}{a^{2} \hbar^{2}}=1$ (thin line), $b / \kappa=-20$ and $\frac{m_{0} \kappa}{a^{2} \hbar^{2}}=1$ (dashed line), $b / \kappa=-20$ and $\frac{m_{0} \kappa}{a^{2} \hbar^{2}}=0.002$ (dotted line).

- Case 4: $m(x)=m_{0} / \cosh (a x)^{4}$

Here $m_{0}$ is a constant mass and $a$ a real parameter. This mass function depicts a solitonic profile and presents a form familiar in effective models of condensed matter and low energy nuclear physics [32]. It has been also used to describe variable diffractions coefficient in nonlinear media [63]. Then the choice

$$
U(x)=\frac{b}{\cosh ^{2}(a x)}
$$

results in the following potential

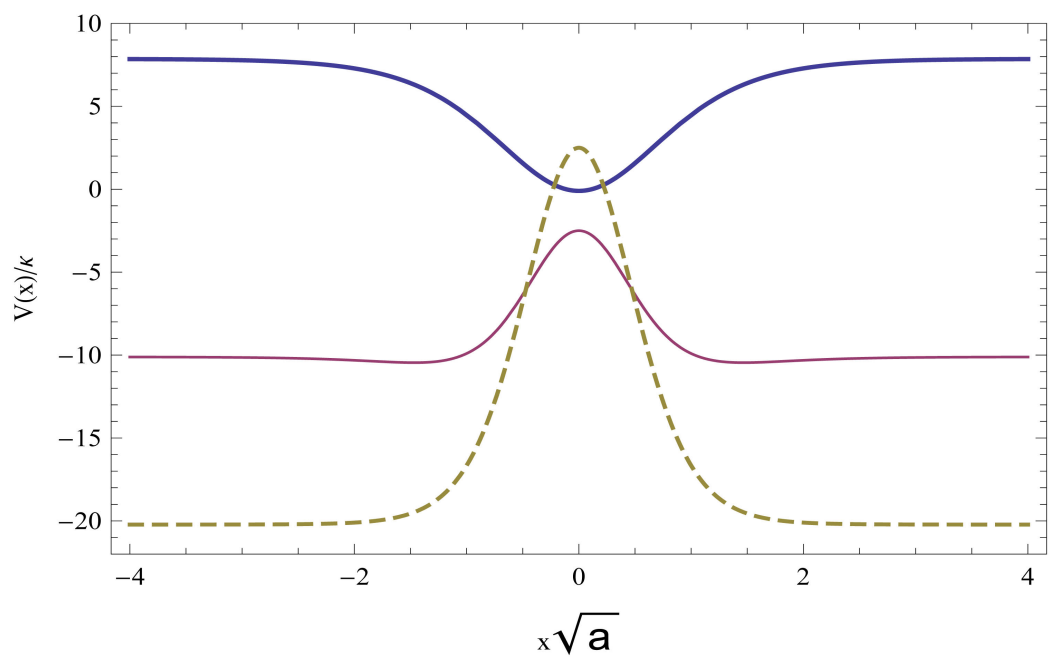

Figure 5. The potential (30) in units of $\kappa$, as a function of $\sqrt{a} x$ for $b / \kappa=0.2$ and $\frac{m_{0} \kappa}{a^{2} \hbar^{2}}=8$ (thick line), $b / \kappa=52$ and $\frac{m_{0} \kappa}{a^{2} \hbar^{2}}=1$ (thin line), $b / \kappa=-50$ and $\frac{m_{0} \kappa}{a^{2} \hbar^{2}}=2$ (dashed line). 


$$
\begin{array}{r}
V(x)=\frac{-b}{2 \cosh ^{2}(a x)}+\frac{m_{0} \tanh ^{2}(a x)}{2 a^{2} \hbar^{2}}\left[\kappa^{2}-\frac{4 b^{2}}{9}-\frac{4 b^{2}}{9 \cosh ^{2}(a x)}\right. \\
\left.-\frac{b^{2}}{9 \cosh ^{4}(a x)}\right]
\end{array}
$$

This hyperbolic potential yields in general a double well interaction. Such kind of potential allows studying situations involving bound states and particle tunneling through a barrier. In particular for $b=0$, i.e. $U(x)=0$ it reduces to a $\tanh ^{2}(a x)$ potential. In Figure 5 we represent the variations of potential (30) for some illustrative values of its free parameters [64].

\section{Conclusion}

To sum up, this work presents a new class of exactly solvable models, endowed with a position-dependent mass, exhibiting a harmonicoscillator-like spectrum. This class has been generated on the basis of a new approach, recently developed by us for the quantum operator relative to the classical non-relativistic kinetic energy, and using the formalism of supersymmetric quantum mechanics. The latter involves factorizing the corresponding Hamiltonian in terms of intertwining operators, along with the requirement of the shape invariance condition. Several concrete examples of PDM systems belonging to this class were discussed.

\section{Acknowledgements}

The authors gratefully acknowledge Qassim University, represented by the Deanship of Scientific Research, on the material support for this research under the number (1671-ALRASSCAC-2016-1-12-S) during the academic year $1437 \mathrm{AH} / 2016 \mathrm{AD}$.

\section{Conflicts of Interest}

The authors declare no conflicts of interest regarding the publication of this paper.

\section{References}

[1] Geller, M. and Kohn, W. (1993) Quantum Mechanics of Electrons in Crystals with Graded Composition. Physical Review Letters, 70, 3103. https://doi.org/10.1103/PhysRevLett.70.3103

[2] Ring, P. and Schuck, P. (1980) The Nuclear Many-Body Problem. Springer, Berlin.

[3] Ganguly, A., Kuru, S., Negro, J. and Nieto, L. (2006) A Study of the Bound States for Square Potential Wells with PositionDependent Mass. Physics Letters A, 360, 228-233. https://doi.org/10.1016/j.physleta.2006.08.032

[4] Plastino, A., Casas, M. and Plastino, A. (2001) Bohmian Quantum Theory of Motion for Particles with Position-Dependent Effective Mass. Physics Letters A, 281, 297-304. https://doi.org/10.1016/S0375-9601(01)00143-8 
[5] de Saavedra, F., Boronat, J., Polls, A. and Fabrocini, A. (1994) Effective Mass of One ${ }^{4} \mathrm{He}$ Atom in Liquid ${ }^{3} \mathrm{He}$. Physical Review B, 50, 4248(R). https://doi.org/10.1103/PhysRevB.50.4248

[6] Puente, A., Serra, L. and Casas, M. (1994) Dipole Excitation of Na Clusters with a Non-Local Energy Density Functional. Zeitschrift für Physik D Atoms, Molecules and Clusters, 31, 283286. https://doi.org/10.1007/BF01445008

[7] BenDaniel, D. and Duke, C. (1966) Space-Charge Effects on Electron Tunneling. Physical Review, 152, 683. https://doi.org/10.1103/PhysRev.152.683

[8] Li, M.T. and Kuhn, K. (1993) Band-Offset Ratio Dependence on the Effective-Mass Hamiltonian Based on a Modified Profile of the GaAs- $\mathrm{Al}_{x}-\mathrm{Ga}_{1-x}$ As Quantum Well. Physical Review B, 47, Article ID: 12760 . https://doi.org/10.1103/PhysRevB.47.12760

[9] Bastard, G. (1981) Superlattice Band Structure in the EnvelopeFunction Approximation. Physical Review B, 24, 5693. https://doi.org/10.1103/PhysRevB.24.5693

[10] Gora, T. and Williams, F. (1969) Theory of Electronic States and Transport in Graded Mixed Semiconductors. Physical Review, 177, 1179. https://doi.org/10.1103/PhysRev.177.1179

[11] Zhu, Q. and Kroemer, H. (1983) Interface Connection Rules for Effective-Mass Wave Functions at an Abrupt Heterojunction between Two Different Semiconductors. Physical Review B, 27, 3519. https://doi.org/10.1103/PhysRevB.27.3519

[12] Morrow, R. and Brownstein, K. (1984) Model Effective-Mass Hamiltonians for Abrupt Heterojunctions and the Associated Wave-Function-Matching Conditions. Physical Review B, 30, 678. https://doi.org/10.1103/PhysRevB.30.678

[13] Bastard, G. (1992) Wave Mechanics Applied to Semiconductor Hetero Structures. EDP Sciences. Les Editions de Physique, Les Ulis, France.

[14] Von Roos, O. (1983) Position-Dependent Effective Masses in Semiconductor Theory. Physical Review B, 27, 7547. https://doi.org/10.1103/PhysRevB.27.7547

[15] Von Roos, O. (1985) Position-Dependent Effective Masses in Semiconductor Theory. II. Physical Review B, 31, 2294. https://doi.org/10.1103/PhysRevB.31.2294

[16] Morrow, R. (1987) Establishment of an Effective-Mass Hamiltonian for Abrupt Heterojunctions. Physical Review B, 35, 8074. https://doi.org/10.1103/PhysRevB.35.8074

[17] Harrison, P. (2000) Quantum Wells, Wires and Dots: Theoretical and Computational Physics of Semiconductor Nanostructures. Wiley, New York.

[18] Boztosun, I., Bonatsos, D. and Inci, I. (2008) Analytical Solutions of the Bohr Hamiltonian with the Morse Potential. Physical Review C, 77, Article ID: 044302.

https://doi.org/10.1103/PhysRevC.77.044302 
[19] Bonatsos, D., Georgoudis, P., Lenis, D., Minkov, N. and Quesne, C. (2011) Bohr Hamiltonian with a Deformation-Dependent Mass Term for the Davidson Potential. Physical Review C, 83, Article ID: 044321. https://doi.org/10.1103/PhysRevC.83.044321

[20] Hamdouni, Y. (2011) Motion of Position-Dependent Effective Mass as a Damping-Antidamping Process: Application to the Fermi Gas and to the Morse Potential. Journal of Physics A: Mathematical and Theoretical, 44, Article ID: 385301. https://doi.org/10.1088/1751-8113/44/38/385301

[21] Mustafa, O. (2011) Radial Power-Law Position-Dependent Mass: Cylindrical Coordinates, Separability and Spectral Signatures. Journal of Physics A: Mathematical and Theoretical, 44, Article ID: 355303. https://doi.org/10.1088/1751-8113/44/35/355303

[22] Foulkes, W. and Schluter, M. (1990) Pseudopotentials with Position-Dependent Electron Masses. Physical Review B, 42, Article ID: 11505. https://doi.org/10.1103/PhysRevB.42.11505

[23] Barranco, M., Pi, M., Gatica, S., Hernandez, E. and Navarro, J. (1997) Structure and Energetics of Mixed ${ }^{4} \mathrm{He}-{ }^{3} \mathrm{He}$ Drops. Physical Review B, 56, 8997.

https://doi.org/10.1103/PhysRevB.56.8997

[24] Morris, J. (2015) New Scenarios for Classical and Quantum Mechanical Systems with Position Dependent Mass. Quantum Studies: Mathematics and Foundations, 2, 359-370.

https://doi.org/10.1007/s40509-015-0037-7

[25] Dekar, L., Chetouani, L. and Hammann, T. (1998) An Exactly Soluble Schrödinger Equation with Smooth Position-Dependent Mass. Journal of Mathematical Physics, 39, 2551.

https://doi.org/10.1063/1.532407

[26] Plastino, A., Puente, A., Casas, M., Garcias, F. and Plastino, A. (2000) Bound States in Quantum Systems with Position Dependent Effective Masses. Revista Mexicana de Fsica, 46, 78.

[27] Alhaidari, A. (2002) Solutions of the Nonrelativistic Wave Equation with Position-Dependent Effective Mass. Physical Review A, 66, Article ID: 042116.

https://doi.org/10.1103/PhysRevA.66.042116

[28] Yu, J. and Dong, S. (2004) Exactly Solvable Potentials for the Schrödinger Equation with Spatially Dependent Mass. Physics Letters A, 235, 194-198.

https://doi.org/10.1016/j.physleta.2004.03.056

[29] Mustafa, O. and Mazharimousavi, S. (2009) SphericalSeparability of Non-Hermitian Hamiltonians and Pseudo-PTSymmetry. International Journal of Theoretical Physics, 48, 183193. https://doi.org/10.1007/s10773-008-9794-y

[30] Bagchi, B., Banerjee, A., Quesne, C. and Tkachuk, V. (2005) Deformed Shape Invariance and Exactly Solvable Hamiltonians with Position-Dependent Effective Mass. Journal of Physics A: Mathematical and General, 38, 2929.

https://doi.org/10.1088/0305-4470/38/13/008 
[31] de Souza Dutra, A. and Almeida, C. (2000) Exact Solvability of Potentials with Spatially Dependent Effective Masses. Physics Letters A, 275, 25-30.

https://doi.org/10.1016/S0375-9601(00)00533-8

[32] Bagchi, B., Gorain, P., Quesne, C. and Roychoudhury, R. (2004) A General Scheme for the Effective-Mass Schrödinger Equation and the Generation of the Associated Potentials. Modern Physics Letters A, 19, 2765-2775.

https://doi.org/10.1142/S0217732304016123

[33] Levy-Leblond, J. (1995) Position-Dependent Effective Mass and Galilean Invariance. Physical Review A, 52, 1845.

https://doi.org/10.1103/PhysRevA.52.1845

[34] Chetouani, L., Dekar, L. and Hammann, T. (1995) Greens Functions via Path Integrals for Systems with Position-Dependent Masses. Physical Review A, 52, 82.

https://doi.org/10.1103/PhysRevA.52.82

[35] Yung, K. and Yee, J. (1994) Derivation of the Modified Schrödinger Equation for a Particle with a Spatially Varying Mass through Path Integrals. Physical Review A, 50, 104.

https://doi.org/10.1103/PhysRevA.50.104

[36] Rajbongshi, H. (2018) Exact Analytic Solution of PositionDependent Mass Schrödinger Equation. Indian Journal of Physics, 92, 357-367. https://doi.org/10.1007/s12648-017-1108-x

[37] Shewell, J. (1959) On the Formation of Quantum-Mechanical Operators. American Journal of Physics, 27, 16.

https://doi.org/10.1119/1.1934740

[38] Trabelsi A., Madouri F., Merdaci A. and Almatar A. (2013) Classification Scheme for Kinetic Energy Operators with PositionDependent Mass. e-print arXiv: 1302.3963v1

[39] Bender, C. and Milton, K. (1997) Nonperturbative Calculation of Symmetry Breaking in Quantum Field Theory. Physical Review D, 55, R3255(R). https://doi.org/10.1103/PhysRevD.55.R3255

[40] Bender, C. and Boettcher, S. (1998) Real Spectra in NonHermitian Hamiltonians Having PT Symmetry. Physical Review Letters, 80, 5243. https://doi.org/10.1103/PhysRevLett.80.5243

[41] Bender, C., Boettcher, S. and Meisinger, P. (1999) PT-Symmetric Quantum Mechanics. Journal of Mathematical Physics, 40, 2201. https://doi.org/10.1063/1.532860

[42] Bender, C. (1999) The Complex Pendulum. Physics Reports, 315, 27-40. https://doi.org/10.1016/S0370-1573(99)00024-1

[43] Bender, C., Dunne, G.V. and Meisenger, P.N. (1999) Complex Periodic Potentials with Real Band Spectra. Physics Letters A, 252, 272-276. https://doi.org/10.1016/S0375-9601(98)00960-8

[44] Bender, C. and Dunne, G.V. (1999) Large-Order Perturbation Theory for a Non-Hermitian PT-Symmetric Hamiltonian. Journal of Mathematical Physics, 40, 4616. https://doi.org/10.1063/1.532991 
[45] Bender, C., Boettcher, S. and Savage, V.M. (2000) Conjecture on the Interlacing of Zeros in Complex Sturm-Liouville Problems. Journal of Mathematical Physics, 41, 6381. https://doi.org/10.1063/1.1288247

[46] Bender, C., Dunne, G., Meisenger, P. and Simsek, M. (2001) Quantum Complex Hénon-Heiles Potentials. Physics Letters A, 281, 311-316. https://doi.org/10.1016/S0375-9601(01)00146-3

[47] Bender, C., Berry, M., Meisenger, P., Savage, V. and Simsek, M. (2001) Complex WKB Analysis of Energy-Level Degeneracies of Non-Hermitian Hamiltonians. Journal of Physics A: Mathematical and General, 34, L31.

https://doi.org/10.1088/0305-4470/34/6/101

[48] Chargui, Y., Dhahbi, A. and Trabelsi, A., A Novel Approach for Constructing Kinetic Energy Operators with Position Dependent Mass. Submitted for publication.

[49] Hassanabadi, H., Chung, W.S., Zare, S. and Alimohammadi, M. (2017) Scattering of Position-Dependent Mass Schrödinger Equation with Delta Potential. The European Physical Journal Plus, 132, 135. https://doi.org/10.1140/epjp/i2017-11422-0

[50] Abramowitz, M. and Stegun, I.A. (1972) Handbook of Mathematical Functions with Formulas, Graphs, and Mathematical Tables. U.S. Government Printing Office.

[51] Carinena, J.F., Ranada, M.F. and Santander, M. (2007) A Quantum Exactly Solvable Non-Linear Oscillator with Quasi-Harmonic Behaviour. Annals of Physics, 322, 434-459. https://doi.org/10.1016/j.aop.2006.03.005

[52] Midya, B. and Roy, B. (2009) A Generalized Quantum Nonlinear Oscillator. Journal of Physics A: Mathematical and Theoretical, 42, Article ID: 285301. https://doi.org/10.1088/1751-8113/42/28/285301

[53] Mostafazadeh, A. (2002) Pseudo-Hermiticity versus PT Symmetry: The Necessary Condition for the Reality of the Spectrum of a Non-Hermitian Hamiltonian. Journal of Mathematical Physics, 43, 205. https://doi.org/10.1063/1.1418246

[54] Mostafazadeh, A. (2002) Pseudo-Hermiticity versus PTSymmetry. II. A Complete Characterization of Non-Hermitian Hamiltonians with a Real Spectrum. Journal of Mathematical Physics, 43, 2814. https://doi.org/10.1063/1.1461427

[55] Mostafazadeh, A. (2002) Pseudo-Hermiticity versus PTSymmetry III: Equivalence of Pseudo-Hermiticity and the Presence of Antilinear Symmetries. Journal of Mathematical Physics, 43, 3944. https://doi.org/10.1063/1.1489072

[56] Kretschmer, R. and Szymanowski, L. (2004) Quasi-Hermiticity in Infinite-Dimensional Hilbert Spaces. Physics Letters A, 325, 112-117. https://doi.org/10.1016/j.physleta.2004.03.044

[57] Cooper, F., Khare, A. and Sukhatme, U. (1995) Supersymmetry and Quantum Mechanics. Physics Reports, 251, 267-385. https://doi.org/10.1016/0370-1573(94)00080-M 
[58] Zhao, F.Q., Liang, X.X. and Ban, S.L. (2003) Influence of the Spatially Dependent Effective Mass on Bound Polarons in Finite Parabolic Quantum Wells. The European Physical Journal B, 33, 3-8. https://doi.org/10.1140/epjb/e2003-00134-3

[59] Mathews, P.M. and Lakshmanan, M. (1975) A QuantumMechanically Solvable Nonpolynomial Lagrangian with VelocityDependent Interaction. Il Nuovo Cimento A, 26, 299-316. https://doi.org/10.1007/BF02769015

[60] Karthiga, S., Chithiika Ruby, V., Senthilvelan, M. and Lakshmanan, M. (2017) Quantum Solvability of a General Ordered Position Dependent Mass System: Mathews-Lakshmanan Oscillator. Journal of Mathematical Physics, 58, Article ID: 102110. https://doi.org/10.1063/1.5008993

[61] Gönul, B., Gönul, B., Tutcu, D. and Özer, O. (2002) Supersymmetric Approach to Exactly Solvable Systems with PositionDependent Effective Masses. Modern Physics Letters A, 17, 20572066. https://doi.org/10.1142/S0217732302008563

[62] Arda, A. and Sever, R. (2011) Bound State Solution$\mathrm{s}$ of Schrödinger Equation for Generalized Morse Potential with Position-Dependent Mass. Communications in Theoretical Physics, 56, 51. https://doi.org/10.1088/0253-6102/56/1/09

[63] Chen, Y., Yan, Z., Mihalache, D. and Malomed, B.A. (2017) Families of Stable Solitons and Excitations in the PT-Symmetric Nonlinear Schrödinger Equations with Position-Dependent Effective Masses. Scientific Reports, 7, Article No. 1257. https://doi.org/10.1038/s41598-017-01401-3

[64] Xie, Q.-T. (2012) New Quasi-Exactly Solvable Double-Well Potentials. Journal of Physics A: Mathematical and Theoretical, 45, Article ID: 175302.

https://doi.org/10.1088/1751-8113/45/17/175302 